\title{
Santé et médecine hospitalière au Saguenay, 1900-1930
}

\section{Normand Perron}

Volume 55, 1988

Cent cinquante ans d'histoire religieuse au Saguenay-Lac-Saint-Jean (1838-1988)

URI : https://id.erudit.org/iderudit/1006948ar

DOI : https://doi.org/10.7202/1006948ar

Aller au sommaire du numéro

Éditeur(s)

Les Éditions Historia Ecclesiæ Catholicæ Canadensis Inc.

ISSN

0318-6172 (imprimé)

1927-7067 (numérique)

Découvrir la revue

Citer cet article

Perron, N. (1988). Santé et médecine hospitalière au Saguenay, 1900-1930.

Sessions d'étude - Société canadienne d'histoire de l'Église catholique, 55, 75-86.

https://doi.org/10.7202/1006948ar

Tous droits réservés @ Les Éditions Historia Ecclesiæ Catholicæ Canadensis Inc., 1988
Ce document est protégé par la loi sur le droit d'auteur. L'utilisation des services d'Érudit (y compris la reproduction) est assujettie à sa politique d'utilisation que vous pouvez consulter en ligne.

https://apropos.erudit.org/fr/usagers/politique-dutilisation/ 


\title{
Santé et médecine hospitalière au Saguenay, 1900-1930
}

\author{
Normand PERRON, historien
}

En 1903, le Progrès du Saguenay, un hebdomadaire qui circule dans la région du Saguenay-Lac-Saint-Jean, relate un grave accident de travail survenu à la Compagnie de pulpe de Chicoutimi et le transport de la victime à son domicile, où elle décède deux heures plus tard, sans qu'il soit jugé opportun de la conduire à l'hôpital local. Une vingtaine d'années plus tard, l'hôpital de Chicoutimi accueille annuellement 300 à 400 accidentés du travail. Contraste important, mais un fait qui, parmi d'autres, illustre l'éclosion de la médecine hospitalière au Saguenay. L'hôpital, qui, jusque vers 1910, avait surtout été utilisé pour l'hébergement d'orphelins, de vieillards et de déshérités, devient peu à peu un centre où des malades reçoivent des soins.

Ce changement, qui survient à Chicoutimi à l'aube du $\mathrm{XX}^{\mathrm{e}}$ siècle, marque l'arrivée de la médecine hospitalière, un phénomène qui a déjà des racines vieilles d'au moins un demi-siècle en Amérique. En bref, le concept de la médecine hospitalière peut se résumer simplement en l'habitude prise par les médecins de soigner à l'hôpital. Mais les exigences du milieu hospitalier se font aussitôt différentes. Toujours plus d'équipements, de locaux, de lits, de personnels spécialisés, voilà en d'autres termes ce que signifie aussi le phénomène de la médecine hospitalière.

La naissance d'un «nouvel» hôpital au Saguenay relève de différents facteurs ayant trait aux conditions socio-économiques, aux perceptions de la santé et de la maladie et, enfin, aux intervenants face au changement. Ces facteurs sont apparus décisifs dans la transformation profonde de l'Hôtel-Dieu Saint-Vallier de Chicoutimi au cours des années 1900-1930. Un bref rappel des origines de cet hôpital permettra, dans un premier temps, de préciser la nature de l'institution au XIX $\mathrm{X}^{\mathrm{e}}$ siècle; dans un deuxième temps, ce rappel aidera à mieux saisir l'ampleur des changements après 1900 . 


\section{L'hôpital de Chicoutimi au XIX'e siècle}

Après sa nomination comme évêque en 1878, Mgr Dominique Racine poursuit, avec encore plus de vigueur, son but de donner au nouveau diocèse de Chicoutimi les institutions indispensables. Parmi elles, l'hôtelDieu $^{1}$, une institution sise à l'ombre des églises et des cathédrales depuis des siècles, le préoccupe plus particulièrement dès 1879 . À ses yeux, le diocèse a besoin de cette institution de charité et d'évangélisation.

Pour des raisons financières, Racine demande d'abord à son ami Hector-Louis Langevin, ministre fédéral des Travaux publics, la construction d'un hôpital maritime. Il évoque l'activité portuaire et, en particulier, l'obligation de loger des matelots malades d'origine, de religion et de culture étrangères dans les familles catholiques de la ville. En 1884, cet hôpital, connu sous le vocable d' «Hôpital de Marine» ouvre ses portes. Entre-temps, l'évêque avait obtenu de le confier aux religieuses augustines de la Miséricorde de Jésus, ce qui lui permettait, du même coup, d'en faire un hôtel-Dieu. Ainsi est né l'Hôtel-Dieu Saint-Vallier.

Avec sept ou huit lits, l'hôpital offre des possibilités bien limitées. Bientôt, les augustines ajoutent des constructions, avec ou sans l'aide de l'État, tantôt sur leurs terrains, tantôt sur ceux du gouvernement. Après la mort de Racine en 1888 et la fin politique de Langevin en 1891, d'autres hommes ont pris la relève. L'arrangement fondé sur l'amitié RacineLangevin se révèle dans toute sa fragilité. Les religieuses ont bientôt le choix de quitter l'hôpital ou de l'acheter. Elles l'acquièrent, en 1895, après des négociations difficiles.

Qui vient à l'hôpital à cette époque? Invariablement, d'une année à l'autre, une dizaine de matelots, quelques ecclésiastiques, des élèves du séminaire, des «pauvres» (vieillards, démunis, etc.), et, à partir de 1894, avec l'ouverture de l'orphelinat, un nombre croissant d'orphelines. Généralement, peu de malades sont hospitalisés et lorsque la population chicoutimienne réclame les services des augustines, la voie du dispensaire lui semble la meilleure. Sur le plan des soins, cet hôpital, comme beaucoup d'autres, souffre d'une mauvaise réputation. À la fin du XIX ${ }^{\mathrm{e}}$ siècle, les Chicoutimiens et les Saguenéens n'y ont guère confiance.

Dans les faits, l'hôtel-Dieu de Chicoutimi fait davantage fonction d'hôpital général jusqu'à la première décennie du $\mathrm{XX}^{\mathrm{e}}$ siècle. Même que les sœurs augustines qui l'ont fondé proviennent de l'Hôpital-Général de Québec. L'Hôtel-Dieu Saint-Vallier sert plutôt à héberger qu'à hospitaliser.

1 Sur les origines de l'Hôtel-Dieu Saint-Vallier, voir Normand PERRON, Un siècle de vie hospitalière au Québec. Les augustines et l'Hôtel-Dieu Saint-Vallier. 1884-1984, Sillery, Presses de l’Université du Québec, 1984, xxiv, 439 p. 
Aussi, dès l'obtention de l'entière propriété de l'institution, les augustines placent-elles en priorité, parmi leurs autres projets, la construction d'un orphelinat. Celui-ci voit d'ailleurs le jour en 1902-1903.

\section{Le milieu socio-économique au début du $\mathrm{XX}^{\mathrm{e}}$ siècle}

Sur un plan temporel, l'implantation de la médecine hospitalière à l'Hôtel-Dieu Saint-Vallier correspond à une époque de changements majeurs du milieu socio-économique régional et, en particulier, du milieu chicoutimien. La transformation de l'hôpital des augustines s'inscrit peutêtre bien dans un courant de modernité.

Les activités agricoles et les activités forestières liées à la coupe et au sciage du bois ont dominé essentiellement l'économie régionale jusqu'à la fin du XIXe siècle. Ces activités agro-forestières contribuent à l'étalement de la population. En 1900, Chicoutimi, la seule municipalité qui a, depuis 1878 , un statut de ville, compte un peu moins de 4000 habitants.

La dispersion de la population limite d'ailleurs la clientèle de l'HôtelDieu. Les utilisateurs peuvent venir de loin pour les cas d'hébergement à long terme, mais ils ne se déplacent pas sur de longues distances pour recevoir des soins à Chicoutimi. La visite du médecin à domicile demeure, de loin, la formule préférée. Aussi, en rapport avec la transformation de l'hôpital, le milieu chicoutimien présente, dans les premières décennies du $\mathrm{XX}^{\mathrm{e}}$ siècle, plus d'intérêt que celui de la région.

Après une éclipse au profit de Roberval dans les années 1880,

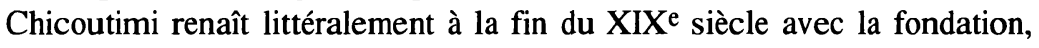
en 1896, de la Compagnie de pulpe ${ }^{2}$. Pour la première fois au Saguenay-Lac-Saint-Jean, une industrie amène une concentration d'emplois au cœur même d'une municipalité. La Compagnie de pulpe aura, de plus, un effet d'entrainement important sur d'autres industries de la ville. La population chicoutimienne croît régulièrement au cours de cette période pour atteindre, en 1930, 12000 habitants. Si l'on y ajoute les populations voisines de Sainte-Anne (Chicoutimi-Nord) et de Rivière-duMoulin, c'est plus de 14000 habitants que compte la région immédiate de l'hôpital.

Les risques d'accidents du travail dans l'industrie de la pulpe et du bois, en général, sont nombreux. Les dirigeantes de l'hôpital établissent, surtout après 1910, un lien entre la présence de la pulperie et la fréquentation de leur institution. Si, par la difficulté qu'il présente, le transport d'un

2 Sur la ville de Chicoutimi, voir Russel BOUCHARD et Normand PERRON, Chicoutimi: la formation de la métropole régionale, Société historique du Saguenay, Cahiers de Saguenayensia, Histoire de Municipalités, $\mathrm{n}^{\circ}$ 4, 1988, 78 p. 
blessé d'un chantier à l'hôpital élimine à toutes fins utiles le recours aux soins hospitaliers, il en va autrement dans le cas d'un accident survenu à la pulperie, celle-ci étant située à moins de deux kilomètres de l'hôpital. Néanmoins, la seule distance à franchir n'explique pas la fréquentation de l'hôpital par les accidentés du travail, puisqu'à la fin des années 1920, les hommes de chantier se rendront à l'hôpital.

Outre l'activité industrielle, le secteur des services contribue au développement de l'économie et de la société chicoutimienne. À titre de ville épiscopale, Chicoutimi continue d'attirer des communautés reiigieuses et elle accapare les principales maisons d'éducation de la région. Au séminaire, qui existe depuis 1873, s'ajoute, en 1907, l'École normale des soeurs du Bon-Pasteur, la première et la seule de la région jusqu'en 1925. Par le biais de l'hôpital, la ville possède aussi l'unique orphelinat pour filles de la région. En 1910, l'École des arts et manufactures voit le jour, grâce aux maristes et à l'Académie qu'ils avaient fondée en 1902. C'est aussi la ville des services financiers. La Banque canadienne nationale établit une succursale en 1894. En 1911, la première caisse populaire de la région ouvre ses portes à Chicoutimi, dans le quartier ouvrier du Bassin.

Enfin, ce milieu socio-économique en effervescence bénéficie d'un relatif isolement géographique. C'est un facteur positif quand il s'agit de fondation et de développement d'institution. Comparativement aux régions à proximité des grands centres urbains, le Saguenay doit se pourvoir d'institutions indispensables à son développement. Ces institutions en régions isolées peuvent alors prospérer parallèlement aux institutions des grands centres.

Le processus d'industrialisation et d'urbanisation crée des conditions propices au développement de la médecine hospitalière à Chicoutimi. Cette ville, par sa prospérité économique et la distance qui la sépare de Québec, présente un terrain favorable à l'éclosion de la médecine hospitalière dès la décennie 1900. Le seul milieu urbain et industriel ainsi que la géographie ne suffisent néanmoins pas à expliquer la transformation de l'hôpital. Toutefois, chose certaine, les religieuses auront choisi de monter dans le train du progrès et de vivre l'esprit de compétition qui caractérise la société chicoutimienne au tournant du XX $\mathrm{XX}^{\mathrm{e}}$ siècle.

\section{Évolution des perceptions de la santé et de la maladie}

Sur le plan de la santé, le désir de dominer la maladie trouve, depuis les XVIII et $\mathrm{XIX}^{\mathrm{e}}$ siècles, des échos nombreux allant des découvertes des Pasteur, Koch et autres jusqu'aux préoccupations sanitaires dans la construction des aqueducs et des égouts. Dans le Québec de la fin du XIXe et du début $\mathrm{XX}^{\mathrm{e}}$ siècle, un virage culturel important touche le milieu de la 
santé (lois, campagnes d'hygiène, sécurité dans les manufactures, environnement). Il résulte des interventions du pouvoir politique et du pouvoir médical, mais aussi de la technologie, de la publicité et des perceptions à l'égard de la santé et de la maladie.

Tout au cours de l'histoire, le pouvoir politique a prôné diverses législations sur la santé et le bien-être. Certaines lois ont attaqué les racines du mal. D'autres mesures politiques ont eu plutôt comme but de corriger les effets du mal. La législation concernant l'accès aux soins hospitaliers s'inscrit évidemment dans cette dernière perspective. Pour la période 1900-1930, les lois québécoises sur les accidents du travail (1909 et 1926) et sur l'assistance publique (1921) sont ici considérées.

Les lois sur les accidents du travail défendent d'abord le droit des ouvriers à recevoir les soins pour les blessures subies en milieu de travail. Le peu de libéralité sur le paiement des soins dispensés aux accidentés du travail limitent toutefois les dispositions de la loi de 1909. Les correctifs apportés en 1926 faciliteront l'accès aux soins hospitaliers, la loi prévoyant les obligations financières de l'employeur à l'égard d'un accidenté du travail. Si les augustines avaient remarqué, vers 1910, les effets de l'activité industrielle sur la fréquentation de l'hôpital, rien de comparable avec la fin des années 1920 où les victimes d'accident du travail monopolisent une partie de l'hôpital ${ }^{3}$. La direction de l'Hôtel-Dieu Saint-Vallier impute d'ailleurs la fermeture de son orphelinat, en 1926, à l'obligation de répondre à la demande de soins. À cette époque, beaucoup de travailleurs blessés viennent des chantiers; la distance n'est apparemment plus un obstacle.

Quant à la loi de l'assistance publique, elle s'adresse d'abord aux utilisateurs. Les subventions accordées aux institutions le sont par le biais d'un simple article 4 de la loi. En facilitant l'accès à l'hôpital à la clientèle démunie, la loi tend à favoriser une utilisation plus large de l'institution hospitalière en cas de maladie. L'esprit de la loi confirmerait la pratique de soigner à l'hôpital. Mais cette loi, c'est aussi la reconnaissance tacite que la santé, par l'accès aux soins, devient un droit pour tous.

Dans une étude des perceptions de la santé et de la maladie, les manifestations du pouvoir médical apparaissent parmi les plus riches de signification. Qu'il s'agisse de l'ensemble des médecins plutôt que des seuls

${ }^{3}$ N. PERRON, Un siècle de vie hospitalière [...], p. 95ss.

${ }^{4}$ L'article 17 de la Loi établissant le service de l'assistance publique prévoit que «Le lieutenant-gouverneur en conseil peut, néanmoins, sur recommandation du service de l'assistance publique, dans les cas d'urgence et de nécessité absolue, aider de la façon qu'il le juge à propos le développement des œuvres d'assistance publique de la province». (Statuts de Québec, 11 George 5, 1921, chapitre 79). 
médecins d'hôpitaux n'a ici guère d'incidence. Le pouvoir médical modifie les perceptions de la santé et de la maladie, ce qui entraînera des conséquences sur l'hôpital.

La fondation de la Société médicale des comtés de Chicoutimi et du Lac-Saint-Jean, en 1900, procure en quelque sorte aux médecins un outil pour affirmer l'importance sociale, politique et médicale de leur profession. En effet, au-delà de l'intervention purement médicale, les médecins se heurtent à de fortes réticences sur le plan social ${ }^{5}$. Une fois leur importance reconnue, les mécecins croieni qu'ils pourroni sensibiliser plus facilement la population et l'État aux problèmes de santé. Aussi, la Société médicale se préoccupe-t-elle de donner à ses membres le prestige scientifique qui devrait leur assurer une emprise sur la population. Une auréole de secret doit entourer la pratique médicale dans le but de renforcer le pouvoir du médecin, particulièrement en médecine curative. Aux médecins, il est recommandé de ne pas divulguer des informations sur la nature de la maladie, sur le médicament utilisé et sur son contenu. Réduit à sa plus simple expression, ce comportement évoque l'ascendant que doit conserver le magicien-sorcier. C'est la ligne de conduite à suivre pour mettre fin au dédain dont sont l'objet le médecin et la profession médicale.

Le corps médical adopte toutefois une attitude différente en ce qui regarde l'aspect préventif de son intervention. Ainsi, les connaissances sur l'hygiène sont diffusées sans restrictions apparentes, comme si les médecins voyaient déjà suffisamment de résistance culturelle à l'acquisition de bons principes d'hygiène. Globalement, sur le plan de la santé préventive, la société régionale présente certains comportements à la fois retardataires et normaux, en regard des progrès médicaux récents. Les croyances et les habitudes évoluent lentement. Les démêlés des médecins avec la population au sujet du respect de la loi dans les cas de maladies épidémiques et de morts suspectes en sont des témoins. Même des curés négligent de respecter la loi sur les certificats de décès et n'encouragent nullement leurs ouailles à s'y conformer. Il se pourrait que la honte et l'esprit d'indépendance aient été des facteurs de résistance importants.

Dans l'ensemble, les médecins du Saguenay-Lac-Saint-Jean travaillent formellement, au cours des années 1900-1930, à obtenir la reconnaissance sociale et politique. À la veille de la Crise, l'objectif semble atteint, même si le corps médical n'est pas parvenu à éliminer totalement du champ des soins les charlatans, les sages-femmes, les religieuses, etc.

Les pouvoirs politiques et médicaux manifestent des engagements nouveaux à l'égard de la santé à une époque où différents progrès et inno-

5 Normand PERRON, «Pour la reconnaissance de la profession de médecin, 19001930», Saguenayensia, 26 (juillet-septembre 1984), p. 86-89. 
vations techniques font reculer les frontières de la nature. La pasteurisation, les découvertes de micro-organismes infectieux, les vaccins, donnent un sentiment de maitrise sur la vie et la nature. La mise au point de l'appareil radiologique en Allemagne, en 1895, permet d'aller au-delà des limites naturelles de l'observation et du diagnostic. Cet appareil captive la direction de l'Hôtel-Dieu Saint-Vallier au point qu'elle songe à acquérir cet instrument de travail dès 1903. Même s'il n'est acheté qu'en 1922, essentiellement pour des raisons financières, l'appareil radiologique symbolise la voie de l'avenir pour la direction de l'hôpital.

Les interventions chirurgicales n'ont rien de nouveau en soi, mais elles sont maintenant définitivement passées des mains des barbiers-chirurgiens et des apprentis-sorciers à celles des médecins. Au Saguenay, les médecins de l'Hôtel-Dieu Saint-Vallier bénéficient d'une première salle d'opération en 1905. Ce n'est toutefois qu'en 1913 que la pièce mal éclairée, mal aérée et située au soubassement de l'hôpital fait place à une salle d'opération moderne. L'ouverture de la nouvelle salle d'opération marque un pas décisif de l'engagement de l'hôpital dans la voie de la médecine hospitalière. Et pour faciliter l'accès aux soins en milieu hospitalier, l'Hôtel-Dieu Saint-Vallier avait inauguré, l'année précédente, un service ambulancier.

L'hôpital, cet endroit si souvent identifié à la mort, devient le lieu de prédilection de la science médicale. Se rendre à l'hôpital signifie bientôt accroître ses chances de guérison. Et avec ce nouvel hôpital, un nouveau médecin naît: le médecin d'hôpital. Celui-ci fera aussitôt sentir sa présence sur le milieu de travail en hôpital: il a besoin de locaux, d'équipement, de personnel.

Les progrès techniques et l'accessibilité à ceux-ci contribuent à modifier l'image que les individus et la société se font de la maladie. La science a lentement raison des connaissances et des croyances populaires. L'affrontement entre la médecine scientifique, d'une part, et la médecine populaire et l'exploitation des croyances culturelles, d'autre part, donnera lieu à des résultats spectaculaires sur les plans de la littérature publicitaire et de la littérature romanesque.

Au Saguenay, la publicité envahit soudainement, vers 1900, le champ de la santé et du bien-être ${ }^{6}$, comme si tout à coup elle pouvait prévoir une écoute favorable aux messages véhiculés dans la presse locale (Le Progrès du Saguenay). La publicité se permet tous les artifices. Utilisant le prestige de la recherche scientifique et recourant à des techniques hardies, elle joue pleinement la corde culturelle dans ses campagnes vantant les vertus médi-

6 Normand PERRON, «Santé, maladie et publicité au Saguenay, 1900-1910», Saguenayensia, 26 (juillet-septembre 1984), p. 102-109. 
cinales des produits pharmaceutiques et autres comme la bière, le vin, les spiritueux.

C'est une publicité importante en quantité, allant de la carte d'affaires au publi-reportage. Elle devient abondante vers 1900 et sa diminution à certaines périodes s'explique par les politiques du Progrès du Saguenay. La publicité occupe alors trop de place dans les pages du journal. De plus, la publicité sur les boissons alcoolisées subit les contrecoups des campagnes antialcooliques.

L'aspect qualitatif de cette publicité instruit davantage sur les changements en cours à propos de la santé et de la maladie. Le point le plus intéressant de cette publicité paraît être la tentative d'intégrer les valeurs culturelles dominantes aux bienfaits des produits de la science moderne. Elle exploite la fragilité de l'enfant, la santé dans la vieillesse, la crédibilité des communautés religieuses, la peur de l'hôpital, la croyance en l'audelà, la négligence coupable et ses conséquences sur l'âme et le corps, pour vendre des produits miraculeux. Cette publicité met encore en relief la dimension que l'homme peut prévenir la maladie. Elle présente aussi la maladie comme une réalité pouvant être autre que celle de la soumission à la nature, que celle de la punition de Dieu. Elle souligne encore la possibilité d'intervenir non seulement sur la maladie, mais aussi de la prévenir.

L'efficacité réelle de toute cette publicité demeure difficile à évaluer. La diversité des annonces publicitaires et la durée de certains produits (Baby's Own, Pilules rouges, vins et spiritueux) laissent toutefois croire à une réponse acceptable.

Écrit en 1913, le roman Maria Chapdelaine de Louis Hémon corrobore cette offensive des valeurs culturelles sur la nature. Plus qu'un roman, ce récit du Canada français apparaît à l'histoire comme une véritable œuvre ethnographique. Maria Chapdelaine, c'est aussi, en effet, l'histoire des perceptions de la santé et du traitement de la maladie. Ici transparait tout le jeu de la nature et des valeurs culturelles ${ }^{7}$. La vie y est présentée en quelque sorte comme un phénomène cyclique, soumise à la loi implacable de la nature. Elle est aussi présentée comme l'enjeu de deux systèmes de valeurs qu'incarnent principalement les personnages de la mère Chapdelaine et sa fille Maria, donc les anciens et les jeunes.

Les personnages de Maria Chapdelaine expriment, à vrai dire, une pluralité de croyances. Les plus âgés rationalisent leur impuissance devant la maladie et la mort par le biais des forces surnaturelles. Chez la jeune génération, le rêve de maîtriser la nature trouve un terrain de prédilection.

7 Normand PERRON, «Perception de la maladie et de la mort dans Maria Chapdelaine», Saguenayensia, 27 (octobre-décembre, 1985), p. 128-131. (Communication présentée au congrès de l'Acfas, 1985.) 
Le cas de Lorenzo Surprenant est instructif à cet égard. Ces jeunes trahissent un sentiment de révolte contre la passivité, contre le destin.

De même, les passages concernant la mort de la mère Chapdelaine sont révélateurs sur le mélange des rites anciens et des croyances modernes: rebouteur contre médecin, connaissance populaire contre connaissance scientifique. Les attitudes à propos de la douleur révèlent des comportements modernes: les intervenants veulent l'éliminer dans la mesure du possible. Les personnages de Maria Chapdelaine donnent aux pilules les vertus presque miraculeuses que véhicule la publicité. Les pilules d'Eutrope Gagnon ont quasiment les propriétés multiples des populaires pilules rouges.

Si le recours à l'hôpital demeure hors de question pour les Chapdelaine, c'est que la distance le rend pratiquement inaccessible. La société décrite dans Maria Chapdelaine n'en présente pas moins un intérêt marqué pour les soins selon des méthodes modernes.

Préoccupations sociales et économiques, intérêts pour les innovations techniques, offensive des médecins, produits pharmaceutiques, tout cela entraîne des attitudes moins soumises envers la maladie et la mort et des changements culturels importants dans les premières décennies du $\mathrm{XX}^{\mathrm{e}}$ siècle. La qualité de la santé devient aussi une question de culture. Les effets se feront sentir, entre autres sur le milieu hospitalier.

\section{Les augustines, les médecins et l'État face au défi de la médecine hospitalière}

Durant les années 1900-1930, dans le cas de l'hôpital de Chicoutimi, les intervenants se limitent essentiellement aux sœurs hospitalières, au corps médical et à l'État. Plus tard, après 1940, le groupe des utilisateurs deviendra, à son tour, un intervenant remarqué, réclamant sans cesse plus de services. Mais c'est d'abord aux sœurs augustines et aux médecins que le défi du changement se pose dans les premières décennies de médecine hospitalière. Ce sont ces deux intervenants qui vont décider, dans le quotidien, de la stagnation, de l'avance plus ou moins rapide de la médecine hospitalière.

Jusque vers 1910 , les relations entre les augustines et le ou les médecins autorisés à pratiquer à l'Hôtel-Dieu Saint-Vallier sont réduites à leur plus simple expression. À cette époque, le médecin, dit médecin visiteur, se présente à l'hôpital lorsque les augustines l'appellent pour traiter les cas difficiles. Les plaintes des médecins s'expriment habituellement sous forme de souhaits. Mais après 1910, les changements amorcés en milieu hospitalier vont rapidement bouleverser l'administration de l'hôpital. Pour 
progresser, la gestion de l'hôpital doit évoluer: la bonne marche de la médecine hospitalière en dépend.

À cette époque, le nombre de malades et d'interventions chirurgicales augmente régulièrement. Vers 1900, l'Hôtel-Dieu Saint-Vallier accueille quelques dizaines de malades par année. En 1930, plus de 1500 malades sont hospitalisés, bon an, mal an. La Crise ne freinera que légèrement la croissance. Même chose en ce qui regarde les interventions chirurgicales: 16 en 1910 comparativement à plus de 1000 vers 1930.

Les médecins de l'Hôtel-Dieu ne lésinent par sur leur formation professionnelle. Certains vont se spécialiser aux États-Unis et en France, ce qui favorise l'application immédiate des dernières connaissances médicales à Chicoutimi. Une clientèle croissante à soigner signifie aussi pour les médecins un rôle accru en milieu hospitalier. D'ailleurs, les médecins s'intègrent sans retard dans le processus de modernisation de l'hôpital.

En 1910, les médecins désirent nettement, pour la première fois, participer à la gestion de l'Hôtel-Dieu Saint-Vallier. Le sentiment que le milieu hospitalier leur appartient les pousse à s'y installer davantage. Désireux d'obtenir la reconnaissance de leur statut dans la nouvelle institution hospitalière, ils décident unilatéralement de former un bureau médical qui se composera des membres actuels et qui devra donner son assentiment pour l'admission des membres futurs. Ils pourront ainsi limiter la venue de nouveaux médecins à l'hôpital. Ils se réservent aussi le droit d'hospitaliser et de traiter les malades. Les augustines demanderont l'intervention de l'abbé Eugène Lapointe qui reconnaît à la communauté son indépendance, son droit de régir son service médical et celui de choisir les médecins. Lapointe n'en conseille pas moins aux religieuses de prendre avis des médecins, un changement notable par rapport au passé.

Avec la loi de l'assistance publique, l'État devient, dans les années 1920, un nouvel intervenant dans le fonctionnement de l'hôpital. Les augustines s'en accommodent: cette loi leur apparaît presque comme un bienfait du ciel, même si elles doivent attendre 1928 avant d'obtenir l'autorisation de demander des subventions gouvernementales. La résistance est venue de l'évêque du diocèse, Mgr Michel-Thomas Labrecque.

L'intervention gouvernementale par le biais de l'assistance publique n'effraie pas trop les augustines. C'est toujours la gestion de l'hôpital qui les préoccupe: sur ce point, les lignes de conduite adoptées avec le gouvernement, avec les corps publics et avec les médecins sont semblables. Ainsi, en 1918, lors de la fondation de l'Hôtel-Dieu de Roberval, les augustines ont dénié tout droit d'intervention à un comité de citoyens désireux de participer à la gestion administrative du nouvel hôpital. En 1928, les sœurs montrent une attitude semblable, cette fois à l'égard des méde- 
cins de Chicoutimi. Elles refusent de reconnaître le comité de médecins qui souhaite intervenir dans l'administration du nouveau pavillon, celui que la communauté s'apprête à construire avec l'aide de l'État. Les médecins de Chicoutimi prétextent l'exemple de l'hôpital Notre-Dame à Montréal, où un comité de médecins participe à l'administration de l'institution. Mais voilà: l'hôpital Notre-Dame n'est pas la propriété d'une communauté religieuse. Par contre, les augustines accepteront que la demande de subvention se fasse au nom de la région plutôt qu'au nom de la communauté, cette procédure n'altérant en rien leur emprise sur la gestion de l'hôpital.

Dans les faits, malgré un refus catégorique de partager la gestion administrative de leur institution, y compris le service médical, l'hôpital s'est engagé pleinement dans la médecine hospitalière, sans trop d'accrocs, puisque les augustines partagent officieusement avec les médecins la gestion médicale de l'hôpital. Les frictions entre les religieuses et le corps médical n'empêcheront pas les médecins de jouer un rôle déterminant dans le développement de la médecine hospitalière et dans la transformation de l'hôpital de Chicoutimi. Les médecins d'hôpitaux, à l'instar de tous les médecins, ont travaillé pour la reconnaissance de leur profession. À l'hôpital, ils seront bientôt les seuls à gérer les soins aux malades.

Pour sa part, l'État, par son aide financière, permet des possibilités nouvelles aux hôpitaux. Agissant sur le long terme, celui-ci évite les susceptibilités en laissant les institutions libres d'adhérer à l'assistance publique. Il se satisfait, dans les débuts, d'une simple surveillance pour celles qui bénéficient de la loi.

Les attitudes des augustines sur l'orientation de l'hôpital montrent une évolution constante à cette époque. La direction opte pour l'hôpital qui soigne des malades plutôt que pour celui qui héberge différents infortunés. Dans les années 1910 et 1920, les décisions d'agrandir et de mieux équiper l'hôpital confirment l'engagement des augustines dans le processus de la médecine hospitalière.

L'ambiance socio-économique chicoutimienne du début du $\mathrm{XX}^{\mathrm{e}}$ siècle a été, certes, un atout favorable au développement de l'Hôtel-Dieu Saint-Vallier. Mais si la prospérité de la ville et l'isolement géographique peuvent stimuler la croissance de l'hôpital, ils n'expliquent pas sa réorientation.

Les perceptions nouvelles de la santé et de la maladie ont des conséquences importantes à l'égard des soins du corps. L'idée de la soumission 
à la maladie recule; celle de la santé perçue comme un droit progresse. L'omniprésence de la maladie et de la mort est en déclin, les progrès techniques aidant. La consommation des soins augmente. Une des manifestations de ce changement culturel transparaîtra dans l'hôpital. Les progrès scientifiques font de celui-ci le lieu idéal de la science médicale dans son combat contre la maladie.

La vision plus matérialiste de la santé, de la maladie et de la mort conduit au déclin de la conception cyclique de la vie pour laisser davantage place à une concepiion linéáaire. Il semble bicn quic le répétitif et la soumission à la nature cèdent le pas, une attitude nouvelle qui pourrait avoir plusieurs conséquences, entre autres sur des activités économiques anciennes comme l'agriculture.

Enfin, la volonté de changement pose aussi le problème du défi au changement. Dans le cas d'un hôpital existant, la principale résistance peut venir du propriétaire. Il y eut effectivement résistance de la part de la communauté des augustines, mais une résistance dirigée sur la gestion administrative de l'hôpital et non contre la progression scientifique de l'institution. Cette résistance a peut-être retardé quelque peu le processus de la médecine hospitalière, mais ne l'a jamais enrayé. Des comparaisons avec des hôpitaux d'autres régions seraient éclairantes. Elles permettraient de déterminer les particularités de l'exemple de Chicoutimi, mais surtout de mieux connaître la période où prend racine l'hôpital moderne au Québec, dont les origines remontent bien avant la Révolution tranquille. 